Public Health

\section{Community-acquired MRSA:}

\section{a practitioner's guide}

Published at www.cmaj.ca on June 27, 2006.

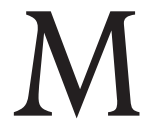
ethicillin-resistant Staphylococcus aureus (MRSA) is usually considered a hospital-acquired (HA) organism. As highlighted elsewhere in this issue of $C M A J$, infections with communityacquired (CA) strains of MRSA are being noted more frequently. The molecular and antimicrobial resistance profiles of these CA-MRSA strains are distinct from HA-MRSA strains. Practitioners must be prepared to diagnose, treat and help prevent these infections. This brief review addresses issues relevant to the identification, prevention and management of CA-MRSA infections for Canadian practitioners.

MRSA comprises the $S$. aureus strains that are resistant to all $\beta$-lactam antimicrobials, including penicillins, cephalosporins and monobactams. A part of the normal flora of humans, $S$. aureus colonizes the anterior nares. It is also an important human pathogen that causes a broad spectrum of infections, from trivial to life-threatening.

CA-MRSA is not new to Canada; it has been endemic in northern aboriginal communities on the prairies for almost 20 years. But the national epidemiology is changing, mirroring that reported in the United States. ${ }^{1}$ Although CA-MRSA infections in Canada have previously been uncommon in nonaboriginal communities, these

Box 1: Infections commonly caused by community-acquired nontoxigenic Staphylococcus aureus

- Boils (furuncles) - Folliculitis

- Cellulitis - Impetigo

- Endocarditis - Osteomyelitis

- Pneumonia, empyema

- Pyomyositis, fasciitis

- Septic arthritis or bursitis

- Wound infection strains are increasingly being isolated from infections in marginalized populations such as incarcerated or homeless people and parenteral drug users (Box I)..$^{2,3}$ In other countries, these bacterial strains have moved into the general population, a trend that also appears to be taking place in Canada. ${ }^{4}$ It seems reasonable to anticipate that MRSA will ultimately replace MSSA (methicillin-sensitive $S$. aureus) in CA $S$. aureus infections over the next 10-20 years, in a process similar to what happened in the rg6os when susceptible $S$. aureus was replaced by penicillinresistant $S$. aureus in hospitals and then in the community.

Although there is some overlap between HA- and CA-MRSA strains, the current CA-MRSA strains generally remain more susceptible to classes of antimicrobials other than $\beta$-lactams, whereas HA strains are more likely to be resistant. The current epidemic strains of CA-MRSA are also characterized by the presence of the Panton-Valentine leukocidin $(p v l)$ gene, which is thought to be a virulence factor for serious skin infections. Management of CA-MRSA involves not only addressing resistance to the usual first-line agents used for the treatment of $S$. aureus infections (i.e., cloxacillin or cephalexin) but recognizing that the CA strains seen nowadays may be more virulent; patients may therefore present with more severe manifestations of infection.

What would make me suspect CAMRSA? Ideally, a practitioner knows whether MRSA is present in the community or not, and uses this information when selecting an initial antimicrobial therapy. Even if CA-MRSA has not yet been reported in a region, patients from high-risk groups or with severe clinical presentations should be considered at risk for CA-MRSA. Patients at high risk include incarcerated or homeless people, parenteral drug users, and any patient with a clinical presentation consistent with $S$. aureus infection who has not responded to initial treatment with a $\beta$-lactam antimicrobial. Infections of particular concern include necrotizing fasciitis, pyomyositis, empyema, severe pneumonia and multiple cutaneous abscesses.

When should cultures be performed? Bacterial specimens for culture do not need to be obtained from all minor skin infections, but any lesion potentially caused by MRSA should be sampled. Culture results not only provide important information to assist with case management, but also help to define the local epidemiology of CA-MRSA strains. As a general rule, a culture should be obtained from any purulent skin lesion that seems serious enough to warrant antimicrobial therapy. Even minor lesions should be cultured if a patient with multiple skin lesions is a member of a recognized high-risk group or if any patient's $\beta$-lactam therapy has failed.

How should a minor $S$. aureus skin infection be managed? One goal of management is to limit antimicrobial therapy where possible. Minor skin lesions in children who are past the neonatal period or in anyone not immunocompromised can usually be managed with local and topical therapy. This includes the use of hot soaks, elevation, and local incision and drainage. Patients should always be instructed in appro-

Table 1: Recommended first line outpatient antimicrobial therapy for communityacquired methicillin-resistant Staphylococcus aureus infection

\begin{tabular}{lll}
\hline Agent & \multicolumn{1}{c}{ Adult patient } & \multicolumn{1}{c}{ Child patient } \\
\hline $\begin{array}{l}\text { Trimethoprim- } \\
\text { sulfamethoxazole } \\
\text { (TMP-SMZ) }\end{array}$ & $\begin{array}{l}\text { One double- }(800 \mathrm{mg} \text { TMP, } 160 \mathrm{mg}) \text { or two regular-strength } \\
(400 / 80 \mathrm{mg}) \text { tablets every } 12 \mathrm{~h}\end{array}$ & $\begin{array}{l}\text { Gauge daily dose at 4-6 } \mathrm{mg} / \mathrm{kg} \\
\text { of patient's body weight for } \\
\text { the TMP component, and divide }\end{array}$ \\
$\begin{array}{ll}\text { Doxycycline* } \\
100 \mathrm{mg} \text { every } 12 \mathrm{~h} \text {, by mouth }\end{array}$ & $\begin{array}{l}\text { For patients } 8 \text { years of age or } \\
\text { older, 100 mg every } 12 \mathrm{~h}\end{array}$ \\
Clindamycin & $\begin{array}{l}150-450 \mathrm{mg} \text { every } 6-8 \mathrm{~h} \text {, by } \\
\text { mouth }\end{array}$ & $\begin{array}{l}\text { Divide } 10-30 \mathrm{mg} / \mathrm{kg} \text { daily for } \\
\text { oral administration every } 6-8 \mathrm{~h}\end{array}$ \\
\hline
\end{tabular}

*Not indicated for patients $<8$ years of age. 
priate wound care, and be advised to seek prompt reassessment if their wound shows signs of deterioration.

How should more serious infections with suspected CA-MRSA be managed? For patients with more severe skin infections, the practitioner should consider whether CA-MRSA could be the cause before prescribing initial empiric therapy. If MRSA is not suspected, then $\beta$-lactam antimicrobials remain the treatment of choice, and are appropriate empiric therapy for mild or uncomplicated infections pending culture results. Where CA-MRSA is considered possible, empiric therapy with a non$\beta$-lactam antimicrobial is reasonable. Agents generally still effective for CAMRSA strains include trimethoprimsulfamethoxazole, tetracycline (doxycycline) or clindamycin ${ }^{1,3}$ (Table I).

Erythromycin-resistant strains may have inducible clindamycin resistance; clindamycin susceptibility should therefore be confirmed with appropriate laboratory testing for erythromycinresistant strains. Linezolid is an effective oral agent, but is not recommended for first-line therapy because our experience with it is limited (especially in children) and its cost is high. Although

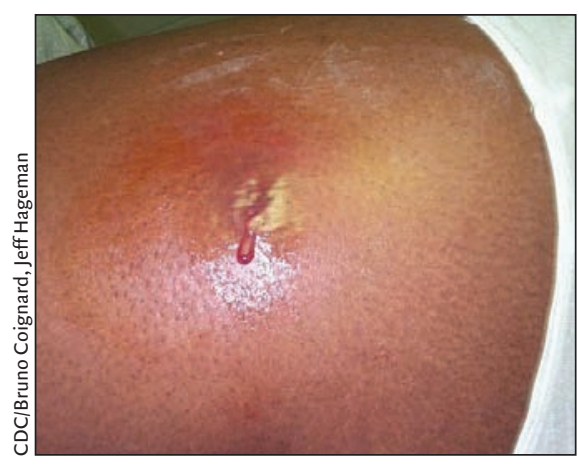

A draining boil caused by a communityacquired strain of methicillin-resistant Staphylococcus aureus, on the upper thigh of a prison inmate.
CA-MRSA strains may be susceptible to fluoroquinolones, these agents are not recommended for first-line therapy because previous experience has repeatedly demonstrated that the development of resistance to fluoroquinolones is rapid when MRSA strains are treated with these agents.

Patients with the most severe clinical presentations of $S$. aureus infection should be managed in the hospital with parenteral therapy.

Other practical considerations: Infection prevention is, of course, preferable to treatment. The basic principles of prevention include careful attention to and constant reinforcement of hand hygiene, not sharing personal-care items (e.g., towels, soap) with others, and early recognition and appropriate containment and management of infections when they do occur (Box 2). All draining wounds should be appropriately covered. These strategies should be applied consistently in settings that are known to be high-risk (e.g., those of sports teams or child daycare), and should be reviewed and implemented in settings where other persons (e.g., family members) may have close, continuing contact with an infected person. Physicians' offices and other health care settings must adhere assiduously to standard precautions, including careful attention to hand hygiene, environmental cleanliness, and appropriate cleaning and sterilization of any equipment for reuse. Additional precautions to prevent CA-MRSA infections are unnecessary in these settings.

Screening for nasopharyngeal carriage or carriage at other sites is not recommended, and decolonization therapy is generally discouraged because of the likelihood of producing even more resistant strains. ${ }^{1}$

CA-MRSA is not currently listed in all provinces as a notifiable disease. If
Box 2: Strategies for preventing transmission of Staphylococcus aureus in the community

- Maintain consistent, appropriate hand hygiene

- Do not share personal items such as razors or towels

- Keep draining wounds covered

- Avoid contact sports, common whirlpools or saunas if a potential participant has an open lesion

- Ensure that communal bathing facilities are clean

an outbreak is suspected, however, public health authorities should be notified to assist in managing the outbreak response, including the investigation of risk factors and interventions for outbreak control.

\section{Lindsay Nicolle}

Departments of Medicine and Medical Microbiology

University of Manitoba

Winnipeg, Man.

\section{REFERENCES}

I. Mulvey MR, MacDougall L, Cholin B, et al; Saskatchewan CA-MRSA Study Group. Communityassociated methicillin-resistant Staphylococcus aureus, Canada. Emerg Infect Dis 2005;1I:844-50.

2. Main CL, Jayaratne P, Haley A, et al. Outbreaks of infection caused by community-acquired methicillin-resistant Staphylococcus aureus in a Canadian correctional facility. Can J Infect Dis Med Microbiol 2005; $6(6): 343-8$.

3. Gilbert M, MacDonald J, Gregson D, et al. Outbreak in Alberta of community-acquired (USA30o) methicillin-resistant Staphylococcus aureus in people with a history of drug use, homelessness or incarceration. CMAJ 2006; I75(2):I49-54.

4. Kurbis CA, Wylie JL. Community-based cluster of methicillin-resistant Staphylococcus aureus in Manitoba. Can J Infect Dis Med Micro 200I;12:I49-52.

\section{ADDITIONAL READING}

- Gorwitz RJ, Jernigan DB, Powers JH, et al. Strategies for clinical management of MRSA in the community: summary of an experts' meeting covered by the CDC. Available: www.cdc.gov/ncidod/dhqp lar_mrsa_ca.html (accessed 2006 June I). 